\title{
Assessment of structural evolution of aggregated soil using neutron tomography
}

\author{
A. Koliji, ${ }^{1}$ P. Lehmann, ${ }^{2}$ L. Vulliet, ${ }^{1}$ L. Laloui, ${ }^{1}$ A. Carminati, ${ }^{3}$ P. Vontobel, ${ }^{4}$ \\ and R. Hassanein ${ }^{4}$ \\ Received 25 June 2007; revised 25 June 2008; accepted 24 July 2008; published 10 October 2008.
}

[1] The advanced nondestructive method of neutron tomography, together with image analysis, is used to evaluate the structural evolution of an aggregated soil during onedimensional compression tests. Aggregation of primary particles is a commonly observed phenomenon in natural and compacted soils that causes an open soil structure with two dominant pore sizes corresponding to macropores (interaggregates) and micropores (intraaggregates). The evolution of macroporosity and the degradation of structures are evaluated by means of morphological parameters such as volume fraction, size distribution, and chord length. Change in the structure is then linked to the macroscopic soil response. It is observed that the major structural modifications are associated with irreversible strains in soil.

Citation: Koliji, A., P. Lehmann, L. Vulliet, L. Laloui, A. Carminati, P. Vontobel, and R. Hassanein (2008), Assessment of structural evolution of aggregated soil using neutron tomography, Water Resour. Res., 44, W00C07, doi:10.1029/2007WR006297.

\section{Introduction}

[2] During the past few years, soil fabric has been studied using various experimental methods such as scanning electron microscopy [e.g., Collins and McGown, 1974; Delage and Lefebvre, 1984], and mercury intrusion porosimetry [e.g., Al-Mukhtar, 1995; Cuisinier and Laloui, 2004]. Among the new approaches, advanced nondestructive methods based on computed tomography (CT) are being increasingly employed in geotechnical engineering [Otani, 2006].

[3] One of these latter methods is neutron radiography and computed tomography. Because of its high sensitivity to the presence of water, this method has been used to study problems involving fluid flow such as fluid infiltration into dry sand [Deinert et al., 2002], the variation of fluid saturation and pore geometry in sandstone samples [Solymar et al., 2003] and, the water flow between soil aggregates [Carminati et al., 2007].

[4] In this study, neutron computed tomography is used as an experimental tool to characterize and quantify the structural evolution of an aggregated soil during an oedometric compression test. The particularity of aggregated soils lies in the fact that they contain porous aggregates which are often separated by large pores. Hence, they are characterized by a double porosity fabric corresponding to micropores (intra-aggregates) and macropores (interaggre-

\footnotetext{
${ }^{1}$ Soil Mechanics Laboratory, Ecole Polytechnique Fédérale de Lausanne, Ecublens, Switzerland.

${ }^{2}$ Laboratory of Soil and Environmental Physics, Ecole Polytechnique Fédérale de Lausanne, Ecublens, Switzerland.

${ }^{3}$ Institute for Terrestrial Ecosystems, Swiss Federal Institute of Technology, Zürich, Switzerland.

${ }^{4}$ Spallation Neutron Source Division, Paul Scherrer Institute, Villigen, Switzerland.

Copyright 2008 by the American Geophysical Union. 0043-1397/08/2007WR006297
}

gates) [Al-Mukhtar, 1995; Delage et al., 1996; Gens et al., 1995; Koliji et al., 2006]. However, the size of aggregates depends on various factors and may differ by several orders of magnitude, from the clay platelets in compacted clays to large aggregates and clods in natural and agricultural soils.

[5] This paper describes the methods, setup and, procedures of the experiments carried out during the course of this study. The presented results allow a better understanding of the relationship between the evolution of soil structure and its macroscopic behavior, and also provide information concerning the mechanisms of structure degradation induced by external mechanical loading.

\section{Materials and Methods}

\subsection{Testing Material and Program}

[6] The tested material is a silty clay from Central Switzerland with a liquid limit, $\mathrm{w}_{1}$, of $38.4 \%$ and a plasticity index, $\mathrm{I}_{\mathrm{p}}$, of $17.7 \%$. After sampling in the field, the soil was gently crushed into smaller parts in its own natural water content of about $7 \%$. The organic parts were carefully removed and aggregates with an average size of $4 \mathrm{~mm}$ were selected by sieving. The obtained aggregates were dried in an oven for $48 \mathrm{~h}$.

[7] The oedometric sample was prepared by free placement of dry aggregates in the cell followed by slight compaction. This method of preparation yielded a normally consolidated sample exhibiting large macropores with a relatively high initial void ratio of 1.63. This sample was then subjected to an oedometric compression test with neutron tomography measurements being done at different loading steps.

\subsection{Neutron Radiography and Tomography}

[8] Neutron radiography is a nondestructive method which, together with image analysis, provides a powerful tool for the experimental study of soil structure [Degueldre et al., 1996]. In this method, a collimated neutron beam is 




Figure 1. Schematic representation of experimental procedure and data processing.

transmitted through the sample (see Figure 1). Neutrons can be absorbed, scattered, or pass undisturbed through the sample. The transmitted neutron beam carries the information about the mass and thickness of the neutron-attenuating components. The intensity of the neutron flux behind the sample is detected by a neutron area detector. The detector is a cooled CCD camera system (DV 434, Andor Technology) looking onto a ${ }^{6} \mathrm{LiF}$ doped $\mathrm{ZnS}$ scintillator screen converting neutron flux into light emission.

[9] Thanks to this system, the radiographs are twodimensional gray value images with values proportional to the transmitted neutron flux. For neutron tomography a set of radiograms is recorded while rotating the sample stepwise by 180 degrees with a constant small angular increment of 0.9 degree. Slices perpendicular to the rotation axis are reconstructed by filtered back projection [Vontobel et al., 2006].

\subsection{Experimental Setup and Procedure}

[10] Figure 1 schematically illustrates the experimental procedure and the followed data processing steps. The sample was first loaded in a loading frame out of the tomography room. To ensure that the cell does not interfere in the beam transfer, a new oedometric cell was built with aluminum (this material is transparent to neutron beams while steel is not). A cylindrical sample of $60 \mathrm{~mm}$ in diameter and $15 \mathrm{~mm}$ in height was subjected to different vertical stress increments. Values of vertical total stress at different steps are given in Table 1. A total of 15 steps including loading and unloading steps were applied, and at each step the vertical deformation of the sample was recorded by direct measurement using a linear variable differential transducer (LVDT).

[11] The macroscopic oedometric response of the sample, namely the total vertical displacement of the sample versus the applied total stress, was deduced from the direct measurements of displacement. After reaching equilibrium at each loading step, the cell was moved from the loading cell to the neutron beam and a tomography of the sample was made. During this step, swelling of the sample in the cell was prevented using a lateral blocking system provided on the piston specifically for this purpose.

[12] Projected image data required preprocessing before they could be used as an input to the tomography reconstruction algorithm. The images had to be normalized for equal neutron exposure and corrected for spatial variations in the incident neutron beam and the inhomogeneity of the scintillator screen (flat field correction). The flat field correction was performed by dividing sample projection images by a radiography image without sample. Moreover, disturbing effects due to neutron scattering were compensated with a correction algorithm given by Hassanein et al. [2005]. After these corrections, computed tomography algorithms yielded the three-dimensional array of the local neutron attenuation coefficient in the sample.

[13] The raw tomography results consisted of a three dimensional sample volume with a voxel (the 3-D equivalent of a 2-D pixel) size of $146 \mu \mathrm{m}$. The reconstructed volumes were processed by a sequence of spatial filters and morphological operations. The processed gray value volume was then used for image analysis and investigation of soil structure changes. The reconstructed volumes of the

Table 1. Loading Steps

\begin{tabular}{|c|c|c|c|c|c|c|c|c|c|c|c|c|c|c|c|}
\hline Step & L0 & $\mathrm{L} 1$ & L2 & L3 & L4 & L5 & L6 & L7 & L8 & L9 & L10 & L11 & L12 & L13 & L14 \\
\hline Vertical total stress $(\mathrm{kPa})$ & 1 & 31 & 195 & 1 & 92 & 195 & 283 & 424 & 1 & 195 & 301 & 424 & 584 & 743 & 1 \\
\hline
\end{tabular}


(a)

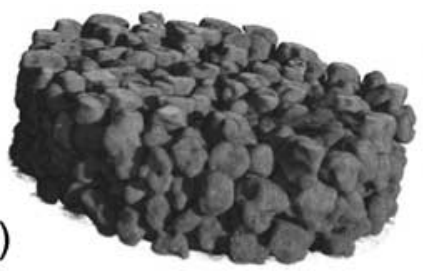

(b)

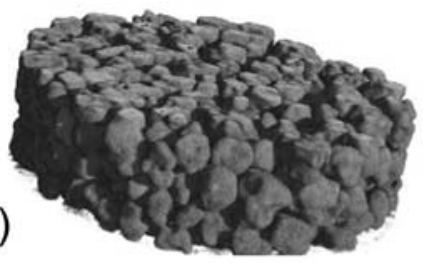

(c)

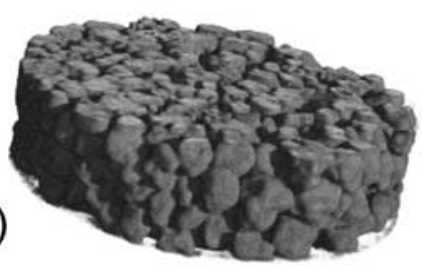

(d)

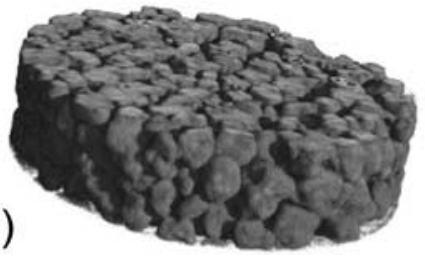


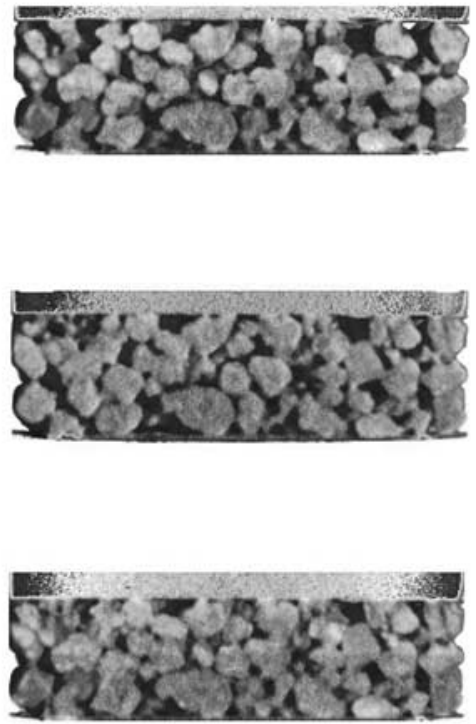

Figure 2. Reconstructed volume and cross section of the sample obtained by neutron tomography at vertical stress of (a) $1.1 \mathrm{kPa}$, (b) $283 \mathrm{kPa}$, (c) $584 \mathrm{kPa}$, and (d) $743 \mathrm{kPa}$. (Initial sample size: $60 \mathrm{~mm}$ in diameter $\times 15 \mathrm{~mm}$ in height).

tomography results are shown in Figure 2 for four different loading steps.

\section{Macroscopic Behavior}

[14] The macroscopic behavior corresponds to the conventional stress-strain response of the soil during the oedometric test. As seen in Figure 3, the oedometric compression curve of the sample exhibits an initial stiff behavior followed by yielding. The initial stiff part of the compression curve is linked to the strength and stiffness of aggregates, namely structural units, which primarily sustain the applied load. The evidence of a similar behavior has been widely reported for natural structured soils [Callisto and Rampello, 2004; Leroueil and Vaughan, 1990; Liu and Carter, 2000].

[15] This phenomenon could be described in the same way as induced overconsolidation; however, the nature of the phenomenon is different. The yield stress is therefore called apparent preconsolidation stress, which is a function of not only stress state and stress history but also the particular structure of the soil structure.

\section{Soil Structure Evolution}

[16] In order to assess the structural evolution of the sample using the tomography data, the concept of double porosity [Barrenblatt, 1963; Warren and Root, 1963] is postulated here. Accordingly, the total porosity, $n$, in aggregated soil reads:

$$
n=n^{\mu}+n^{m}
$$

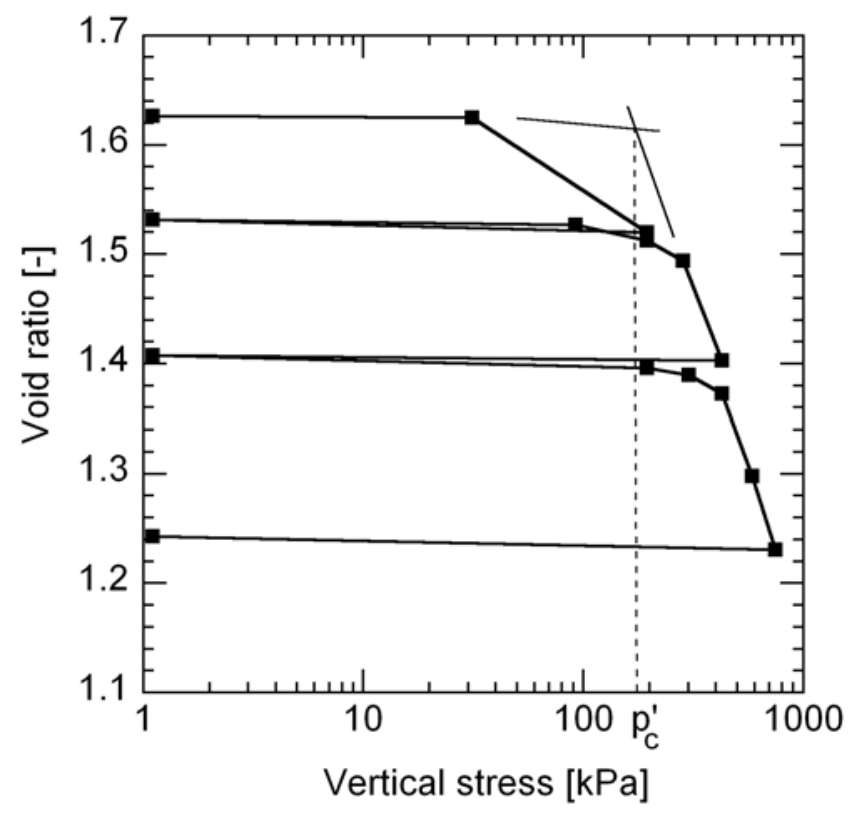

Figure 3. Oedometric curve of the sample. 

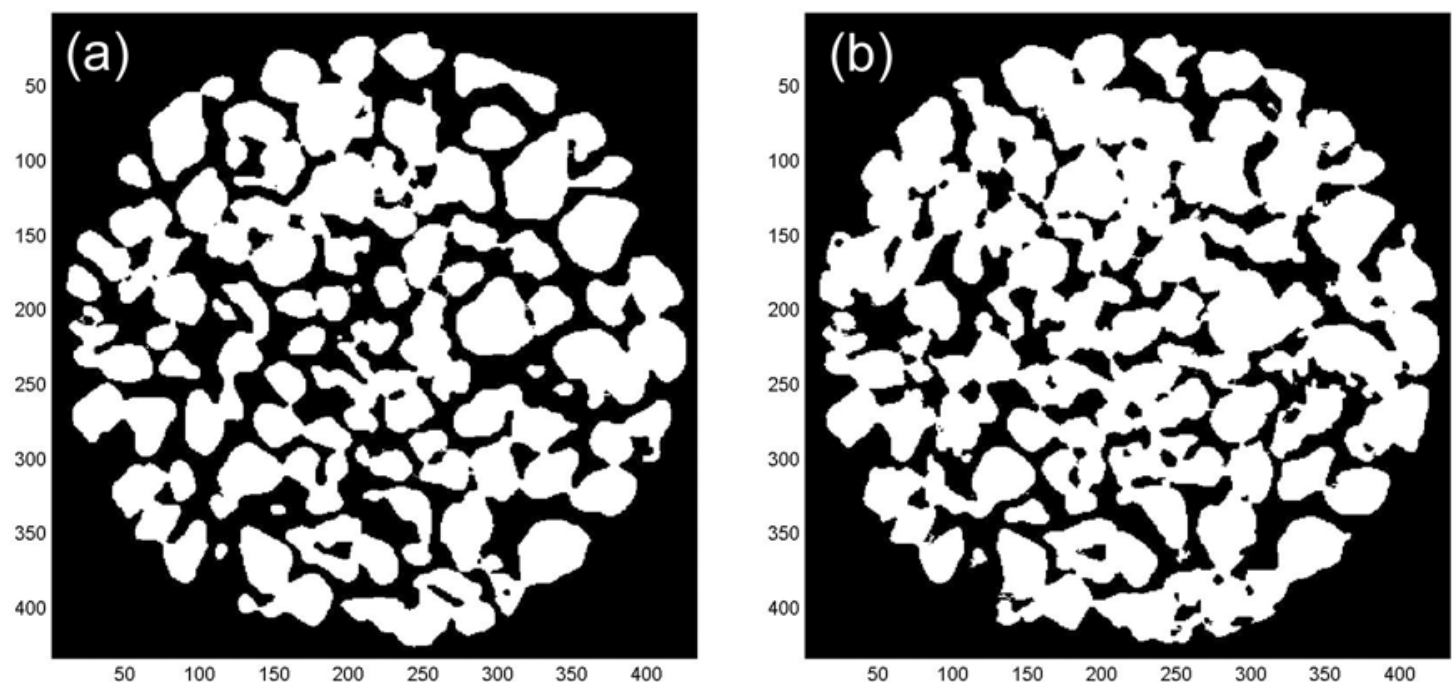

Figure 4. Horizontal cross sections of scanned samples. The neutron transmission images were segmented into aggregates (white) and pores between them (black): (a) initial state, $1.1 \mathrm{kPa}$, (b) final state, $742.7 \mathrm{kPa}$. The numbers denote the coordinates in pixels with pixel size of 146 microns.

where $n^{\mu}$ and $n^{m}$ respectively denote the microporosity corresponding to the intra-aggregates pores and the macroporosity corresponding to the interaggregate pores.

[17] Macroporosity in the aggregates soils is a representative measure of the soil structure for the mechanical analysis, where the main concern is the deformation. Macropores are retained by the aggregated structure; hence, the macroporosity and soil fabric are directly affected by aggregate breakage and degradation of interparticle bonds within the aggregates.

[18] Considering the two components of the soil structure, i.e., soil fabric and interparticle bonding [Mitchell, 1993], and their strong interaction in aggregated soils, macroporosity evolution represents the main structural evolution in these materials. It is noteworthy that other features of soil structure such as connectivity and tortuosity of the pores are not of primary importance for the stress-strain response of the soil, and therefore, are not addressed here.

[19] The macroporosity of the sample at different loading steps is deduced from the tomography results in order to track soil structure evolution during the test. For this purpose, reconstructed tomography volume results are segmented into aggregates (including micropores) and the macropores. This yields a binary volume of the sample in which a distinction is made between aggregates and macropores (Figure 4). Macroporosity at each load step is deduced from this binary volume. Since segmented aggregates represent both solid particles and micropores within the aggregates, microporosity cannot be calculated by an analysis of the segmented images. However, it can be deduced from equation (1), with $n$ and $n^{m}$ being known.

[20] Figure 5 shows the evolution of macroporosity obtained by image analysis as well as total porosity by direct external measurements. The two curves follow similar evolution patterns and differ mainly in magnitude. However, for a given stress increment, the relative decrease of macroporosity is slightly more than the corresponding decrease in total porosity. This evidence suggests that, in the applied range of stress values, the main deformation is due to a decrease in the volume fraction of macropores (due to volume change closure or infilling). This observation is in agreement with results coming from an analysis of the pore size distribution of double porous soils during compaction using the mercury intrusion technique [Delage and Lefebvre, 1984; Cuisinier and Laloui, 2004].

[21] The values of macroporosity plotted in Figure 5 are the average values in the whole sample; however, local values of macroporosities during the loading are not necessarily the same in different part of the sample. In order to evaluate the effects of induced external loading in different parts of the sample, macroporosity was analyzed as a function of space (vertical distribution through the sample) and loading history. The sample was divided into horizontal disks of one unit thickness (1 voxel) along its height, and

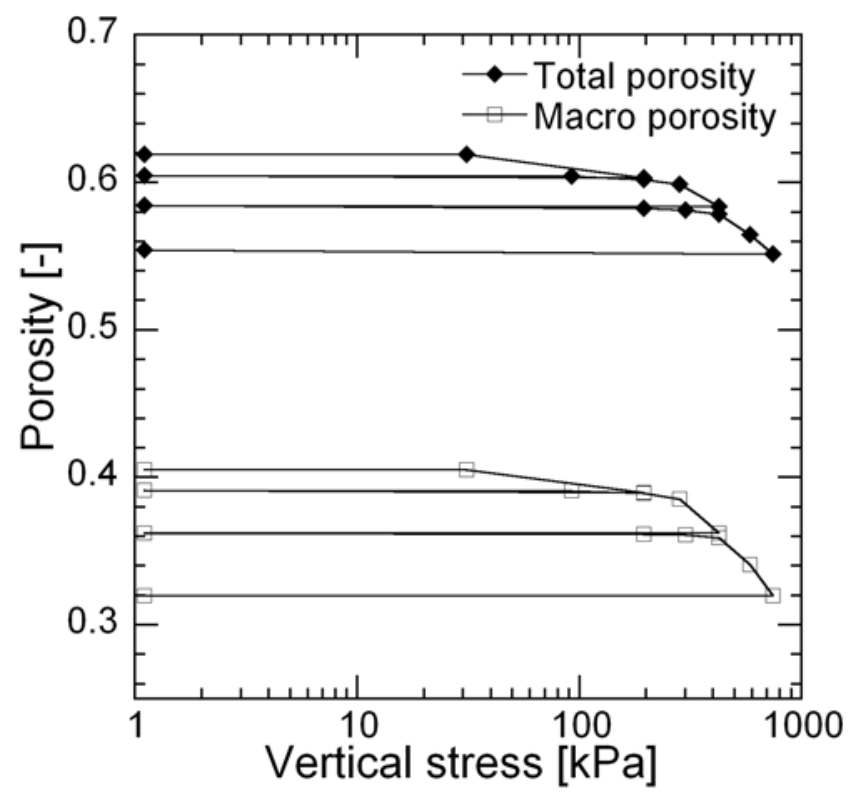

Figure 5. Evolution of total and macroporosity versus applied stress. 

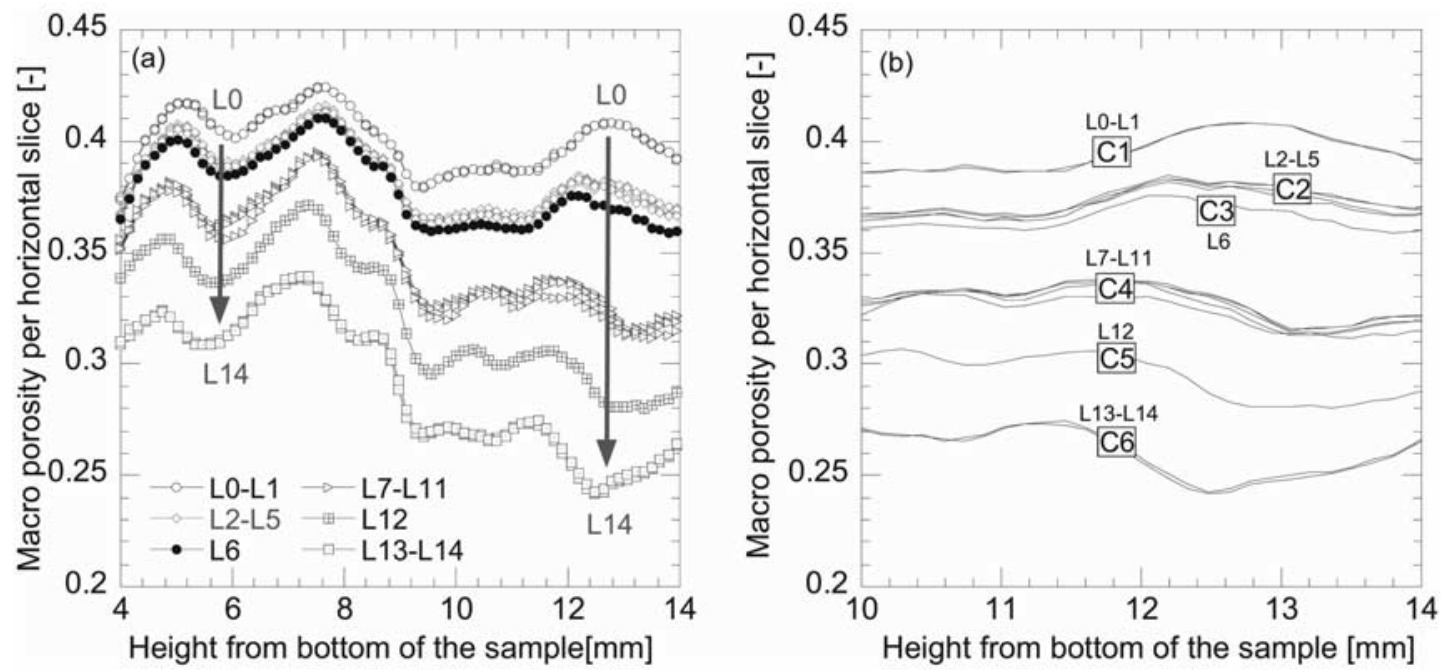

Figure 6. Distribution of macroporosity along the height of the sample for different loading steps.

hence, macroporosity of each single disk represented the value of macroporosity at the given height of the disk in the sample. The results of this analysis are plotted in Figure 6a for different loading steps L0 to L14 (see Table 1). Each curve in Figure 6a represents the distribution of macroporosity along the height of the sample for the given loading step. In order to prevent interference from the boundary effects and height variations in the sample on the analysis, only the middle $10 \mathrm{~cm}$ of the sample was considered. It can be observed that changes in macroporosity are not homogenous in the sample; a more pronounced decrease of macroporosity occurs in the upper part of the sample.

[22] A magnified plot of this upper part is shown in Figure $6 \mathrm{~b}$. An interesting aspect of Figure $6 \mathrm{~b}$ is the fact that the fifteen steps of loading and unloading can be reduced into six main classes indicated by $\mathrm{C} 1$ to $\mathrm{C} 6$. This division classifies the state of the material at different loading steps on the basis of the induced changes in soil structure, such that each class represents a set of loading steps during which no major modification of macroporosity occurs.

[23] Accordingly, the classification of loading steps could be also shown on the oedometric compression curve of the sample as depicted in Figure 7. Figure 7 reveals that the initial states, with an applied stress of less than the initial apparent preconsolidation stress (i.e., apparently overconsolidated states), reside in the same class. Moreover, all of the states situated on an unloading-reloading path belong to a single class. Changes in class are observed only during the normal consolidation state of the sample. This shows that states sharing the same (apparent) preconsolidation stress remain in the same class and therefore they exhibit the same soil structure. Subsequently, changes in the soil structure occur only with changes in preconsolidation stress levels. In other words, plastic strain is associated mainly with changes in macroporosity. This important experimental finding has a major impact on the modeling of this phenomenon.

\section{Mechanism of Structure Degradation}

[24] In the previous section, the evolution of soil structure was linked to the macroscopic description of soil structure through apparent effective preconsolidation stress. However, modeling of the evolution of soil structure due to applied stress (or induced deformation) requires, as a key issue, knowledge of structural degradation mechanisms. This could be achieved through the geometric information of the macropores that reflect changes in the size and shape of macropores, and their relative positions and arrangements in the sample during the loading process. These two issues are considered here for different loading steps. For the sake of simplicity, for further macroporosity analysis, each class (C1 to $\mathrm{C} 6$ ) is denoted only by one single representative loading step.

[25] In order to address the first issue, the evolution of macropore size distribution of the sample is evaluated. The three-dimensional pore size distribution is analyzed by inserting spherical structural elements in the macropore

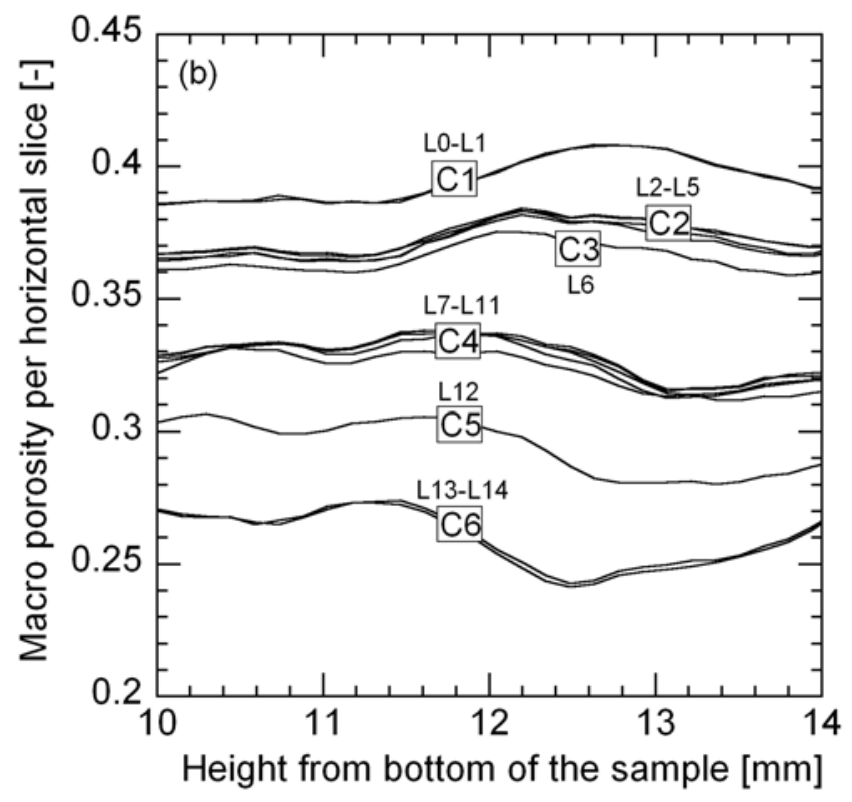

Figure 7. The six structural classes on the oedometric curve. 


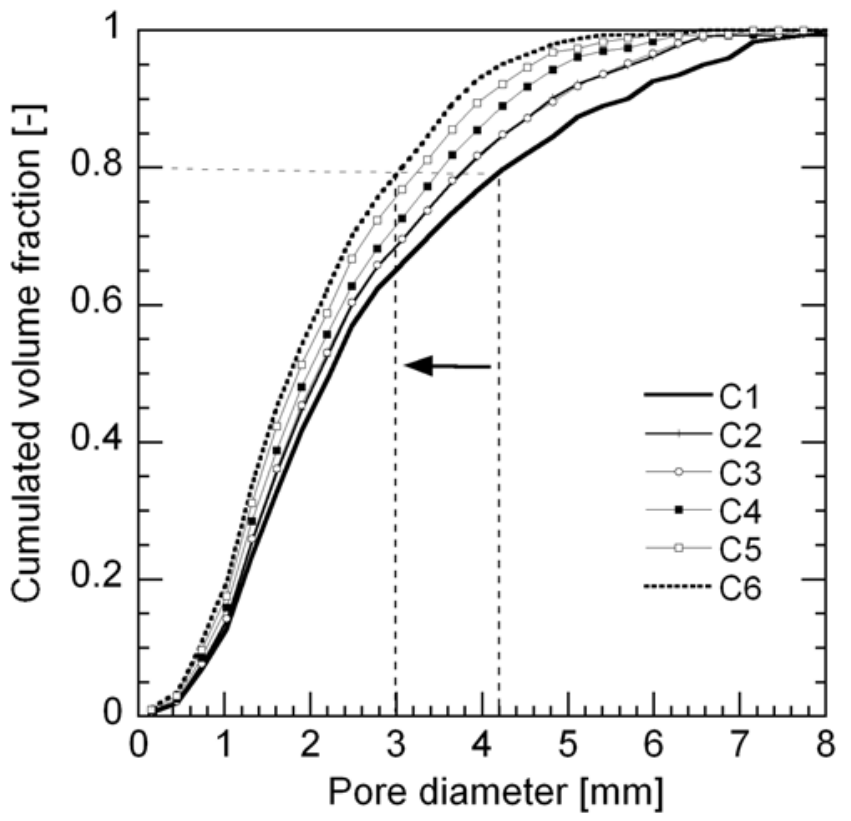

Figure 8. Cumulated macropore size distribution.

space. The cumulated macropore size distribution (MPSD) of the sample is plotted for the six identified classes in Figure 8. It is observed that the pore diameter corresponding to a given cumulated volume fraction decreases as the applied stress increases. This indicates the decrease in the size of macropores.

[26] Three dimensional geometrical analysis of the pore space is required to verify if the change in size is as well associated with the change in shape of macropores. A candidate parameter for this purpose is the chord length distribution. Chords are line segments between the intersections of a line with the interface of aggregates and macropores. In the other word, chord length of a single aggregate in a given direction, is the length of a straight line that could be located within the aggregate in that direction. Accordingly, changes in the chord length of macropores in three dimensions could be used to evaluate the change in pore shape. The chord lengths in the vertical ( $z$ axis) and two perpendicular horizontal directions ( $x$ and $y$ axis) were calculated for segmented volumes of both macropores and aggregates. Chord lengths are plotted versus the corresponding volume fraction of pores (Figures 9a-9c) and aggregates (Figures 9d-9f) for the six classes.

[27] Figures $9 \mathrm{a}-9 \mathrm{f}$ illustrate the decrease in volume fraction of macropores with longer chord lengths and increase in volume of shorter chord lengths with increasing applied stress. This observation was made for all three dimensions. The relative increase of the volume fraction (with respect to initial value) for the most dominant pore radius in the initial state is $49.8,47.8$ and 45.2 percent in $x$, $y$ and $z$ directions, respectively. This indicates that macropores changes in size occur almost isotropically in the three directions; hence, no significant change in the shape of the macropores occurs.

[28] Contrary to the macropores, the shorter chord lengths of aggregates decrease with loading. One interpretation of this observation could be that individual aggregates merge together and therefore, the thickness of the layer of the aggregate space increases.

[29] The second issue to be addressed, in order to understand structural degradation mechanisms, is the relative arrangement and redistribution of macropores in the sample during the loading process. For this purpose, it is important to make a distinction between two modes of destructuring which cause the soil fabric to change (1) local closure of macropores and (2) rearrangement of macropores in the sample.

[30] In the first phenomenon, a change in fabric is mainly due to local changes in the size of the macropores without significant rearrangements of the pores; it is reasonable to associate this mode of degradation with an isotropic loading condition. The second phenomenon has to be linked to distortional deformation which occurs during deviatoric stress paths. The open question is whether the mechanism of structural degradation that results in a structured soil reaching a fully destructured state is isotropic, deviatoric or a combined mechanism of the two.

[31] This fact is investigated here by the analysis of gray value images. In this analysis, the gray value represents the aggregate mass; that is, the higher the gray value in a region, the greater the concentration of aggregate mass. The gray values are averaged as a function of radial distance from the vertical axis. The mean gray value represents the circumferential concentration of the aggregate mass at a given distance from the vertical centerline of the sample. These values are calculated and shown in Figure 10 for the six identified classes. Minima represent regions with less aggregate mass and more pores. Two main observations can be made: as the applied load increases (1) the average gray value across the sample increases, and (2) the relative positions of extrema do not change.

[32] The first observation suggests that at the applied range of stress an important fraction of macropores still exists between the aggregates. This means that state of material is still far from a homogenous state were all the macropores are closed.

[33] The second observation could be interpreted to mean that there is no significant rearrangement of macropores (aggregates) at the current condition of loading, and the relative positions of macropores and aggregates remain almost the same. This evidence indicates that the reduction of macroporosity in the sample is mainly due to the local closure of macropores; therefore, the active mechanism of degradation in the current loading condition is mainly isotropic.

[34] Considering these two pieces of evidence, it is understood that the active isotropic mechanism for structural degradation by itself is not enough to fully remove the aggregated structure of the material. Accordingly, it is concluded that a fully destructured state of material could be achieved only through a combination of both isotropic and deviatoric mechanisms of destruction. More experimental results are required to verify the generality of this observation for different loading conditions and different ranges of applied stress.

\section{Conclusion}

[35] The nondestructive method of neutron tomography together with image analysis was used to evaluate the 

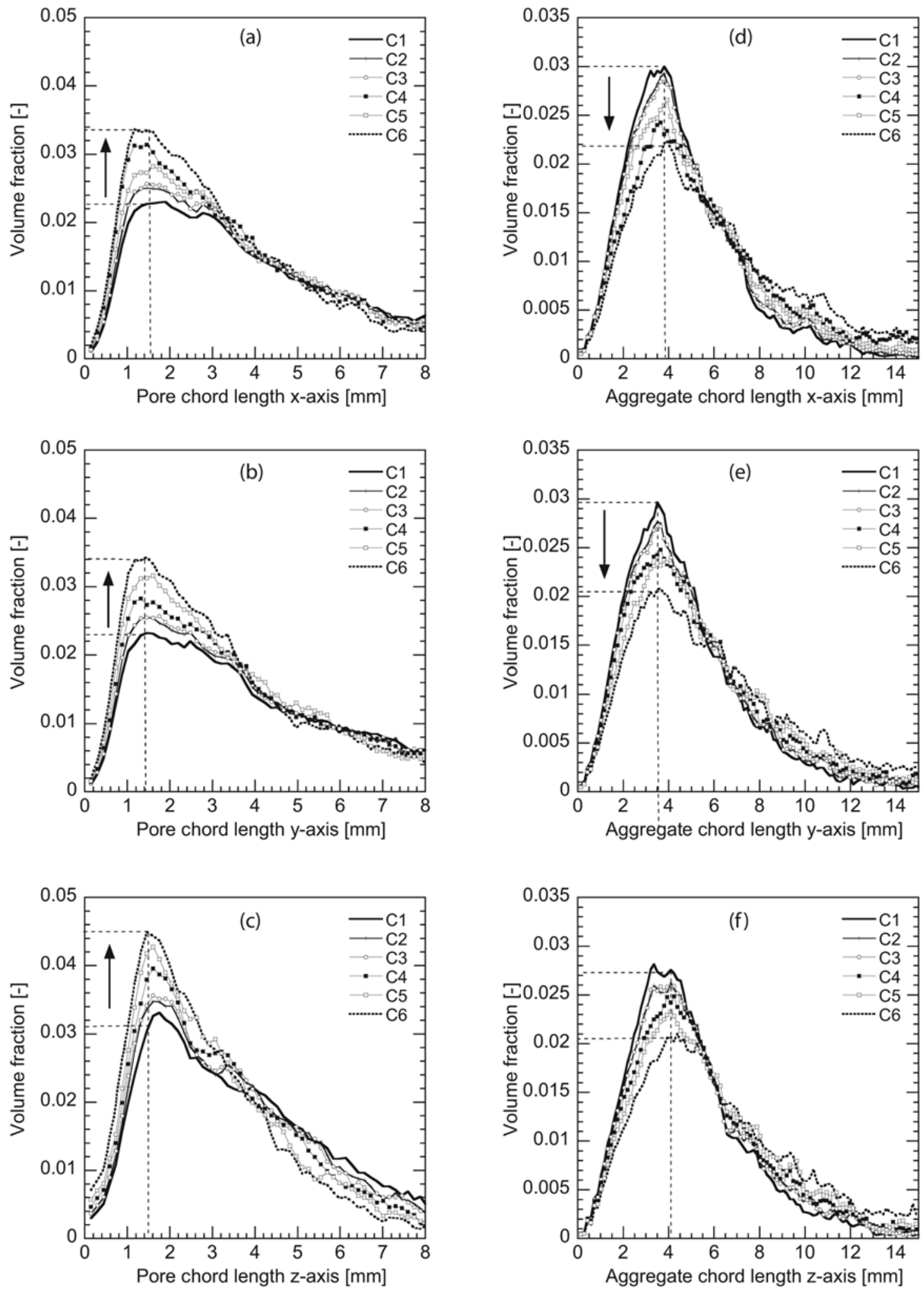

Figure 9. Chord length distribution of macropore space $(a, b, c)$ and aggregates $(d, e, f)$ in the vertical ( $z$ axis) and two perpendicular horizontal ( $x$ and $y$ axis) directions.

structural evolution of an aggregated soil during the oedometric test. The macroscopic oedometric response showed an initial stiff behavior followed by yielding. Although stemming from a different nature, this phenomenon was described in the same way as induced over consolidation.
[36] Tomography results showed that during the mechanical loading, the macropores are essentially affected while the change in the micropores is almost insignificant. Moreover, analysis of the tomography data showed that significant changes in macroporosity occur only during normal 


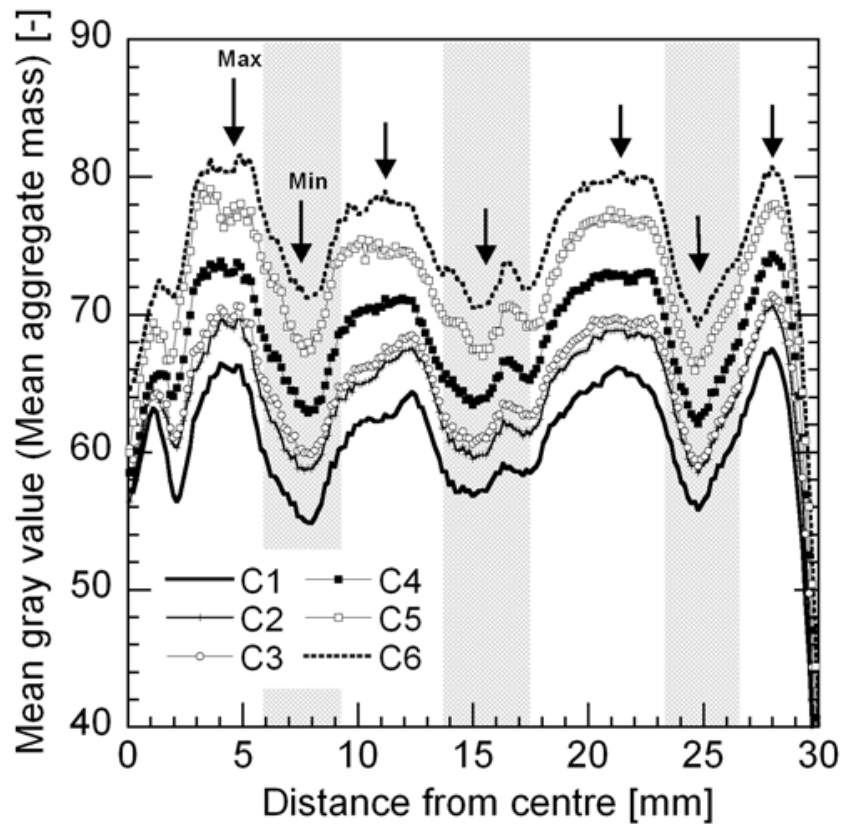

Figure 10. Mean gray value (mean aggregate mass) as a function of distance from the center of the sample.

consolidation compression while, during unloading and reloading steps, no significant change in macroporosity was observed. It is therefore understood that any major structural evolution is associated with plastic strain.

[37] Geometric characterization of macropores and aggregates during the test revealed that isotropic mechanism of degradation alone is not enough to remove the aggregated structure of the soil and bring it to the state of a reconstituted soil. This evidence suggests considering a combination of volumetric and deviatoric plastic strains to describe structural degradation for modeling purposes.

[38] Acknowledgments. The authors wish to thank the partners at ITÖ-ETHZ, H. Flühler and A. Kaestner, as well as E. Lehmann at PSI for the access to the neutron beam facility. This work is financed by the research and prospective service of EPFL.

\section{References}

Al-Mukhtar, M. (1995), Macroscopic behavior and microstructural properties of a kaolinite clay under controlled mechanical and hydraulic state, in Unsaturated Soils: Proceedings of the 1st International Conference on Unsaturated Soils/UNSAT 95, edited by E. E. Alonso and P. Delage, pp. 3-9, Balkema, A.A., Rotterdam, Netherlands.

Barrenblatt, G. I. (1963), On certain boundary value problems for the equation of seepage of liquid in fissured rock, Prikladnaja Mat. Mekhanika, 27, 513-518.

Callisto, L., and S. Rampello (2004), An interpretation of structural degradation for three natural clays, Can. Geotech. J., 41, 392-407, doi:10.1139/t03-099.
Carminati, A., et al. (2007), Water flow between soil aggregates, Transp. Porous Media, 68(2), 219-236, doi:10.1007/s11242-006-9041-z.

Collins, K., and A. McGown (1974), Form and function of microfabric features in a variety of natural soils, Geotechnique, 24(2), 223-254.

Cuisinier, O., and L. Laloui (2004), Fabric evolution during hydromechanical loading of a compacted silt, Int. J. Numer. Anal. Methods Geomech., 28(6), 483-499, doi:10.1002/nag.348.

Degueldre, C., H. Pleinert, P. Maguire, E. Lehmann, J. Missimer, J. Hammer, K. Leenders, H. Böck, and D. Townsend (1996), Porosity and pathway determination in crystalline rock by positron emission tomography and neutron radiography, Earth Planet. Sci. Lett., 140, 213-225, doi:10.1016/ 0012-821X(96)00043-X.

Deinert, M., J.-Y. Parlange, T. S. Steenhuis, K. Unl̈u, J. Selker, and K. B. Cady (2002), Real-time measurement of water profiles in a sand using neutron radiography, paper presented at Hydrology Days, AGU, Fort Collins, Colo., $1-4$ April.

Delage, P., and G. Lefebvre (1984), Study of the structure of a sensitive Champlain clay and of its evolution during consolidation, Can. Geotech. J., 21(1), 21-35.

Delage, P., M. Audiguier, Y.-J. Cui, and M. D. Howat (1996), Microstructure of compacted silt, Can. Geotech. J., 33, 150-158, doi:10.1139/t96030 .

Gens, A., E. E. Alonso, J. Suriol, and A. Lloret (1995), Effect of structure on the volumetric behavior of a compacted soil, in Unsaturated Soils: Proceedings of the 1st International Conference on Unsaturated Soils/ UNSAT 95, edited by E. E. Alonso and P. Delage, pp. 83-88, Balkema, A.A., Rotterdam, Netherlands.

Hassanein, R., E. Lehmann, and P. Vontobel (2005), Methods of scattering corrections for quantitative neutron radiography, Nucl. Instrum. Methods Phys. Res., Sect. A, 542(1-3), 353-360.

Koliji, A., L. Laloui, O. Cusinier, and L. Vulliet (2006), Suction induced effects on the fabric of a structured soil, Transp. Porous Media, 64(2), 261-278, doi:10.1007/s11242-005-3656-3.

Leroueil, S., and P. R. Vaughan (1990), The general and congruent effects of structure in natural soils and weak rocks, Geotechnique, 40(3), 467488.

Liu, M. D., and J. P. Carter (2000), Modelling the destructuring of soils during virgin compression, Geotechnique, 50(4), 479-483.

Mitchell, J. K. (1993), Fundamentals of Soil Behavior, 437 pp., Wiley, New York.

Otani, J. (2006), X-ray computed tomography for geotechnical engineering, in Advances in X-ray Tomography for Geomaterials, edited by J. Desrues, G. Viggiani, and P. Besuelle, pp. 95-115, ISTE, London.

Solymar, M., E. Lehmann, P. Vontobel, and A. Nordlund (2003), Relating variations in water saturation of a sandstone sample to pore geometry by neutron tomography and image analysis of thin sections, Bull. Eng. Geol. Environ., 62, 85-88.

Vontobel, P., E. H. Lehmann, R. Hassanein, and G. Frei (2006), Neutron tomography: Method and applications, Physica B, 385-386, 475-480, doi:10.1016/j.physb.2006.05.252.

Warren, J. R., and P. J. Root (1963), The behaviour of naturally fractured reservoirs, Soc. Pet. Eng., 245-255.

A. Carminati, Institute for Terrestrial Ecosystems, Swiss Federal Institute of Technology, CH-8092 Zürich, Switzerland.

R. Hassanein and P. Vontobel, Spallation Neutron Source Division, Paul Scherrer Institute, CH-5232 Villigen, Switzerland.

A. Koliji, L. Laloui, and L. Vulliet, Soil Mechanics Laboratory, Ecole Polytechnique Fédérale de Lausanne, CH-1015 Ecublens, Switzerland. (azad.koliji@epfl.ch)

P. Lehmann, Laboratory of Soil and Environmental Physics, Ecole Polytechnique Fédérale de Lausanne, CH-1015 Ecublens, Switzerland. 